\title{
The power of language-concordant care: a call to action for medical schools
}

\author{
Rose L. Molina ${ }^{1,2^{*}}$ (D) and Jennifer Kasper ${ }^{2,3,4}$
}

\begin{abstract}
We live in a world of incredible linguistic diversity; nearly 7000 languages are spoken globally and at least 350 are spoken in the United States. Language-concordant care enhances trust between patients and physicians, optimizes health outcomes, and advances health equity for diverse populations. However, historical and contemporary trauma have impaired trust between communities of color, including immigrants with limited English proficiency, and physicians in the U.S. Threats to informed consent among patients with limited English proficiency persist today. Language concordance has been shown to improve care and serves as a window to broader social determinants of health that disproportionately yield worse health outcomes among patients with limited English proficiency. Language concordance is also relevant for medical students engaged in health care around the world. Global health experiences among medical and dental students have quadrupled in the last 30 years. Yet, language proficiency and skills to address cultural aspects of clinical care, research and education are lacking in pre-departure trainings. We call on medical schools to increase opportunities for medical language courses and integrate them into the curriculum with evidence-based teaching strategies, content about health equity, and standardized language assessments. The languages offered should reflect the needs of the patient population both where the medical school is located and where the school is engaged globally. Key content areas should include how to conduct a history and physical exam; relevant health inequities that commonly affect patients who speak different languages; cultural sensitivity and humility, particularly around beliefs and practices that affect health and wellbeing; and how to work in language-discordant encounters with interpreters and other modalities. Rigorous language assessment is necessary to ensure equity in communication before allowing students or physicians to use their language skills in clinical encounters. Lastly, global health activities in medical schools should assess for language needs and competency prior to departure. By professionalizing language competency in medical schools, we can improve patients' trust in individual physicians and the profession as a whole; improve patient safety and health outcomes; and advance health equity for those we care for and collaborate with in the U.S. and around the world.
\end{abstract}

Keywords: Language-concordant care, Health equity, Medical school, Language skills

\section{Background}

This article argues for the importance of languageconcordant care (clinical encounters in which the patient and care provider speak the same native language) and the role of medical schools in providing language courses to support medical students in delivering languageconcordant care. While the ethics around providing meaningful access to language services for all patients through qualified interpreters are critical, we advocate for language

\footnotetext{
* Correspondence: rmolina@bidmc.harvard.edu

${ }^{1}$ Division of Global and Community Health, Department of Obstetrics and Gynecology, Beth Israel Deaconess Medical Center, Boston, MA 02215, USA

${ }^{2}$ Scholars in Medicine Office, Harvard Medical School, Boston 02115, MA, USA Full list of author information is available at the end of the article
}

courses to improve language competency in clinical encounters and appropriate language assessments of providers who identify as being proficient in multiple languages.

\section{Main text \\ "In this work against sickness, we begin not with genetic or cellular interactions, but with human ones. They are what make medicine so complex and fascinating. How each interaction is negotiated can determine whether a doctor is trusted, whether a patient is heard, whether the right diagnosis is made, the right treatment given. But in this realm, there are no perfect formulas.” [1] (p65)}

(c) The Author(s). 2019 Open Access This article is distributed under the terms of the Creative Commons Attribution 4.0 International License (http://creativecommons.org/licenses/by/4.0/), which permits unrestricted use, distribution, and reproduction in any medium, provided you give appropriate credit to the original author(s) and the source, provide a link to the Creative Commons license, and indicate if changes were made. The Creative Commons Public Domain Dedication waiver (http://creativecommons.org/publicdomain/zero/1.0/) applies to the data made available in this article, unless otherwise stated. 
If we could be granted a superpower, many of us would choose omnilingualism - the ability to speak, read, and write all languages. We live in a world of incredible linguistic diversity; nearly 7000 languages are spoken globally and at least 350 are spoken in the United States. Globally, the five most spoken languages are Chinese, English, Hindi, Spanish and Arabic; an estimated 650 million speak English as a second language [2]. According to 2016 U.S. Census data, 25.4 million Americans speak English less than "very well" [3] and the Bureau expects this to remain relatively constant in 2020 [4]. As the U.S. and other countries around the world become more ethnically and culturally diverse, the power to communicate and interact with each other holds even greater importance [5].

In the U.S., language concordance has been vaguely defined as a clinical encounter where the patient and doctor speak the same non-English language [6]. In countries where there is more than one dominant language, such as Canada, scholars have defined language concordance as situations where health care providers and patients speak to each other in their shared native language [7]. Language-discordant encounters occur when patients and health care providers speak different first languages, which may manifest as differences in proficiency and experience and therefore hinder the ability to communicate nuances critical for understanding [7]. Language concordance is a particularly important foundation to gain trust, optimize health outcomes and advance health equity in diverse patient populations. Medical and other health professional schools [8] are optimally positioned to catalyze a deeper sensitivity to language and professionalize language curricula to cultivate skills and behaviors in students who strive for language concordance in clinical encounters. While this debate focuses on literature in the U.S., the challenge of language concordance in clinical care is relevant around the world [5].

The phenomenon of trust and its essential role in human cooperation and competition was investigated experimentally for the first time in the 1950s [9]. Trust is defined as "a judgement by the trustor [e.g. patient], requiring the acceptance of resultant vulnerability and risk, that the trustee (individual or organisation, [e.g. physician]) has the competence, willingness, integrity and capacity (i.e. trustworthiness) to perform a specified task under particular conditions." [10] Applying Deutsch's theory of cooperation and competition to the patient-clinician therapeutic alliance, the process needs to include honesty, confidence, and open communication. Patients' health outcomes depend on their belief that they share valued goals with their physicians. An inability to articulate valued goals or a breach in trust because of a language barrier hence becomes an obstacle to developing a therapeutic alliance and improving health outcomes.

Historical trauma has broken trust between communities of color, including immigrants with limited English proficiency, and physicians. One example is the forced sterilization of Mexican women in Los Angeles county in the late 1960s-1970s. In Madrigal v. Quilligan, working-class migrant women reported they underwent tubal ligation at the time of their cesarean deliveries without their informed consent [10]. While the plaintiffs lost the case, they won important changes to informed consent around sterilization, including the requirement of bilingual consent forms [11].

Threats to informed consent among patients with limited English proficiency persist today. A retrospective review of patients undergoing lumbar puncture, thoracentesis or paracentesis at a hospital with interpreter services found English-speaking patients were almost twice as likely to have full informed consent documentation as those with limited English proficiency [12]. Notably, fewer than half of patients with limited English proficiency had a consent form in their primary language or an English consent form signed by an interpreter [12]. In addition to communication challenges in obtaining informed consent, a systematic review demonstrated that language discordance between patients and physicians diminished patients' sense of integrity and intensified their vulnerability in the clinical setting [13].

In addition to the accurate exchange of information necessary to ensure patient safety, language concordance builds trust in the patient-doctor relationship. While interpreters play important roles in clinical encounters, language-concordant providers are better equipped to build rapport and bond with patients, provide higher quality care, and increase patient satisfaction than languagediscordant providers with in-person or telephone-based interpreters [6]. A study of pediatric patients presenting to an urban emergency room found that, in comparison to English-speakers, Spanish-speaking patients had lower trust of individual physicians and higher mistrust of the medical care they receive in comparison to other ethnic groups [14]. In addition, language was a driver of health disparities; Spanish speakers had lower intervention and hospital admission rates [14]. Meeting a doctor who speaks the same language puts patients at ease by alleviating their anxiety about communication; this fosters a sense of relief and gratitude, which form the basis for a therapeutic alliance.

Language concordance between physicians and patients has been shown to improve care through fewer medical errors, increased understanding of illness and the treatment plan, adherence to the treatment plan, and satisfaction with care [15]. In one observational study of Latinx patients with diabetes, patients were asked to describe how well their personal physician spoke their preferred language without an interpreter. Language-discordant physicians were those who patients reported only spoke "fair," "poorly," or "does not speak my language." By contrast, language-concordant physicians were those who patients reported as speaking 
the preferred language "well," "very well," or "excellent." The study demonstrated that patients with limited English proficiency who had a language-discordant physician had higher odds of poor glycemic control compared to those who had a language-concordant physician, even after controlling for potential demographic and clinical confounders [16]. Communicating health-related information in the appropriate language and literacy level is critical to ensure understanding of and ability to complete treatment plans, and thereby optimize health outcomes $[17,18]$.

Language-concordant care also serves as a window into understanding the broader social determinants of health that disproportionately yield worse health outcomes among patients with limited English proficiency. In the United States, the Patient Protection and Affordable Care Act of 2010 created Accountable Care Organizations (ACOs) and Health Communities (AHCs) to respond to the burgeoning evidence that addressing health-related social needs through enhanced clinical encounters and clinical-community liaisons can improve health outcomes and reduce costs $[19,20]$. Through culturally humble and linguistically appropriate communication, physicians are positioned to discuss sensitive topics such as food and housing insecurity and intimate partner violence; ask about barriers that impede their patients' health; and facilitate connecting their patients to needed resources. Language alone can be an insurmountable barrier that makes navigating other social determinants of health-such as access to safe housing, job security, and transportationnearly impossible. Providing language-concordant care is an opportunity for physicians and care teams to help patients overcome the social factors that impact their health and well-being [21].

In addition to the clinical encounters in the United States, language fluency and humility is relevant for medical students engaged in healthcare around the world. Global health experiences among U.S. medical and dental students have quadrupled in the last 30 years [22, 23]. One-third of graduating medical students report a global health experience. Yet, appropriate training in language proficiency and skills to address cultural aspects of clinical care, research and education are lacking in pre-departure trainings [24]. Rather, most trainings focus primarily on safety and travel logistics, and a much smaller proportion address ethics and cultural humility. A web-based review of U.S. medical schools found that $32(24 \%)$ had a structured global health program, but only one addressed language or cultural proficiency [25]. A recent study of eight undergraduate global health programs found only one that explicitly named language proficiency training as a key course objective [26]. Linguistic disparities can negatively impact research, education, patient care, capacity-building and system strengthening in global settings [27-29]. In response, the World Health Organization (WHO) is leading efforts to bridge language gaps in public health [2]. One example is the "Voices in Global Health" course offered at Duke Global Health Institute, where students speak in a non-English language while learning key global health topics [30]. Given the critical interplay of language, culture, trust, collaboration and ethics in global health settings, now is an opportune time to introduce and enhance language awareness and training in global health courses offered during undergraduate medical education.

To develop a workforce with competency in languageconcordant care, medical schools will need to integrate language courses into the curriculum and provide opportunities for students to maintain and improve their skills as they learn clinical medicine. Currently, there is a relative paucity of options, with the majority of schools focused only on Spanish courses. A national survey of medical schools found that $66 \%$ had a medical Spanish curriculum in place in 2012-2014, and 62\% of those schools reported the medical Spanish curriculum had been in place for at least five years [31]. Additionally, 32\% of medical schools without a Spanish curriculum planned to implement one in the subsequent two years [31]. The most common reasons cited for implementing this type of curriculum were the growing Spanish-speaking patient population and student interest. A variety of teaching strategies were employed, such as didactic instruction, student-to-student role play, standardized patients, and clinical encounters with patients [31]. Additionally, some institutions integrated interpreter shadowing, online modules, and case discussions. However, there was marked variation in the types of assessments done at the end of the courses: few schools used an Objective Structured Clinical Examination or standardized patient evaluation, and only one used a formal language competency assessment from an external company [31].

While these findings demonstrate an interest in providing language courses during medical training, work must be done to professionalize language competency in medical schools before allowing students to use their language skills in clinical encounters. Some recommendations include setting fluency level criteria for curricula and developing modules that can facilitate retention of language skills over time [7]. In fact, the Affordable Care Act Rule 1557 strictly regulates "meaningful access" for patients with limited English proficiency, including qualifications for those who can act as interpreters. Some hospitals have begun to implement language competency assessments for health professionals who self-identify as being proficient in another language. To achieve equity in communication, health professionals need to demonstrate competency in communicating nearly as well in another language as they do in their native language. However, linguistic competency is not the only sine qua non of the patient - provider relationship [32]; other aspects of building a therapeutic alliance should also be intentionally taught and role-modeled in medical education. 
Given the complexity of language barriers spanning from linguisitic proficiency to health literacy to body language and other aspects of communication, it is critical for medical schools to develop curricula through interdisciplinary collaboration with experts in health communication science, linguistics, and social science. To operationalize language concordance in medical education training, feasibility challenges and unintended consequences may arise. Leadership support, financial sustainability, and student interest are critical for success. Additionally, language curricula and competency assessments should be developed with the goal of benchmarking a level of proficiency at which physicians can be trusted to practice safely in that language. Without such an evaluation, graduates may feel ready to use their acquired language skills in clinical encounters despite lacking sufficient competency to communicate effectively. For example, international medical graduates need to demonstrate a level of language proficiency as a prerequisite for providing patient care in that language. In contrast, applying a gold standard of communicating as native speakers may be in conflict with what is reasonable and feasible. It may result in fewer health professionals demonstrating superior linguistic competency for use in clinical settings, which would lead to even greater reliance on interpreters and subsequent delays in timely access to interpreters for clinical care. A rigorous assessment tool for medical linguistic competency that incoporates the content of most clinical interactions, faculty expertise, patient safety, and medical students' other pressing educational needs is needed. Such an assessment is relevant for both primary English-speaking medical students seeking to acquire new language skills and students who already have proficiency in a language other than English.

While language curricula are required to build and assess verbal competency in clinical encounters, the hidden curriculum regarding the use of professional versus ad hoc interpreters must also be addressed. The indifference to ensuring appropriate interpreter services for patients with limited English proficiency needs to be rooted out from the clinical learning environment through positive role modeling and facilitating access to qualified interpreters [33]. In one study, one of every 40 malpractice claims were related, all or in part, to failure to provide appropriate interpreter services [34]. Interpreters in health care settings undergo rigorous training and must follow a strict code of ethics [35]. There is a robust literature on the ethics of using professional rather than ad hoc interpreters (e.g. children, family members, friends, and medical assistants who are not qualified interpreters) in medical care. Most importantly, the use of qualified interpreters results in better and more efficient patient care [34, 36, 37].
Recently, the Association of American Medical Colleges (AAMC) published guidelines about core entrustable professional activities (EPAs) that address the foundational behaviors, tasks and responsibilities in which all graduating medical students need to be proficient for clinical care, patient safety, and to enter residency programs [38]. Interpersonal and communication skills are integrated throughout all of the EPAs, yet there is no explicit guidance around appropriate use of interpreters for patients with limited English proficiency in the AAMC EPA Toolkit [39]. Through these EPAs, there is an opportunity to teach appropriate use of interpreters that challenge the predominant hidden curriculum around language services during every clinical encounter.

\section{Conclusions}

We call on medical schools to increase opportunities for medical language courses with evidence-based teaching strategies, integrated content around health equity, and standardized language assessments. The languages offered should reflect the needs of the patient population both where the medical school is located and where the school is engaged globally. Key content areas should include how to conduct a history and physical exam, relevant health inequities that commonly affect the patients who speak different languages, cultural sensitivity and humility particularly around beliefs and practices that affect health and wellbeing, and how to work in language-discordant encounters with interpreters and other modalities. The pedagogical approaches may vary based on the size of the class and resources, but priority should be placed on interactions with health practitioners, patients and community members who are native speakers and immersion opportunities in clinical and community sites when possible. Rigorous language assessment is necessary to ensure equity in communication before allowing students or physicians to use their language skills in clinical encounters. Lastly, global health activities in medical schools should assess for language needs and competency (e.g. local languages and dialects, student fluency in those languages and dialects, plan for language-discordant situations, and availability of interpreters) prior to departure. By professionalizing language competency in medical schools, we can improve patients' trust in individual physicians and the profession as a whole; improve patient safety and health outcomes; and advance health equity for those we care for and collaborate with in the U.S. and around the world.

\footnotetext{
Abbreviations

AAMC: Association of American Medical Colleges; ACOs: Accountable Care Organizations; AHCs: Accountable Health Communities; EPAs: Entrustable professional activities; WHO: World Health Organization
}

Acknowledgements

We thank Jasmine Stecker for her assistance with reference management and Dr. Jeffrey Katz for reviewing prior versions of this manuscript. 


\section{Authors' contributions}

RLM and JK participated in conception of manuscript, drafting, and critically revising the manuscript. Both authors read and approved the final manuscript.

\section{Funding}

None.

\section{Availability of data and materials Not applicable.}

\section{Ethics approval and consent to participate}

Not applicable.

\section{Consent for publication}

Not applicable.

\section{Competing interests}

The authors declare that they have no competing interests.

\section{Author details}

'Division of Global and Community Health, Department of Obstetrics and Gynecology, Beth Israel Deaconess Medical Center, Boston, MA 02215, USA. ${ }^{2}$ Scholars in Medicine Office, Harvard Medical School, Boston 02115, MA, USA. ${ }^{3}$ Pediatrics and Global Health and Social Medicine, Harvard Medical School, Boston, MA 02115, USA. ${ }^{4}$ Department of Newborn Medicine, Brigham and Women's Hospital, Boston, MA 02115 USA.

Received: 14 April 2019 Accepted: 12 September 2019

\section{Published online: 06 November 2019}

\section{References}

1. Gawande A. Better: a Surgeon's notes on performance. New York, NY: Picador; 2008.

2. World Health Organization. Bridging the language divide in health. Bull World Health Organ. (https://www.who.int/bulletin/volumes/93/6/15-02 0615/en/). 2015;93(6):361-440. Published June 2015. Accessed 9 Jan 2019.

3. United States Census Bureau. Detailed languages spoken at home and ability to speak English for the population 5 years and over: 2009-2013. (https://www.census.gov/data/tables/2013/demo/2009-2013-lang-tables. html). Published Oct 2015

4. Ortman JM, Shin HB. Language projections: 2010 to 2020. (https://www. census.gov/hhes/socdemo/language/data/acs/Ortman_Shin_ASA2011_ paper.pdf). Published Aug 2011.

5. Lindenmeyer A, Redwood S, Griffith L, Teladia Z, Phillimore J. Experiences of primary care professionals providing healthcare to recently arrived migrants: a qualitative study. BMJ Open. 2016;6(9):e012561.

6. Ngo-Metzger Q, Sorkin DH, Phillips RS, et al. Providing high-quality care for limited english proficient patients: the importance of language concordance and interpreter use. J Gen Intern Med. 2007;22(Suppl 2):324-30.

7. Kehayia $E$, Segalowitz N. Exploring the determinants of language barriers in health care ( $\mathrm{LBHC})$ : toward a research agenda for the language sciences. Can Mod Lang Rev. 2011:67(4):480-507.

8. Ali P, Johnson S. Speaking my patient's language: bilingual nurses perspective about provision of language concordant care to patients with limited English proficiency. J Adv Nurs. 2017:43(2):421-32.

9. Deutsch M. Trust and suspicion. J Conflict Resol. 1958;2(4):265-79.

10. Damodaran A, Shulruf B, Jones P. Trust and risk: a model for medical education. Med Educ. 2017:51(9):892-902.

11. Stern AM. STERILIZED in the name of public health - race, immigration, and reproductive control in modern California. Am J Public Health. 2005;95(7):1128-38.

12. Schenker $Y$, Wang F, Selig SJ, Ng R, Fernandez A. The impact of language barriers on documentation of informed consent at a hospital with on-site interpreter services. J Gen Intern Med. 2007;22(S2):294-9.

13. Rocque $R$, Leanza $Y$. A systematic review of patients' experiences in communicating with primary care physicians: intercultural encounters and a balance between vulnerability and integrity. PLoS One. 2015;10(10):e0139577.

14. Fields A, Abraham M, Gaughan J, Haines C, Hoehn KS. Language matters: race, trust, and outcomes in the pediatric emergency department. Pediatr Emerg Care. 2016;32(4):222-6.

15. Green AR, Nze C. Language-based inequity in health care: who is the "poor historian"? AMA J Ethics. 2017;19(3):263-71.
16. Fernandez A, Schillinger D, Warton EM, et al. Language barriers, physicianpatient language concordance, and glycemic control among insured Latinos with diabetes: the diabetes study of northern California (DISTANCE). J Gen Intern Med. 2010;26(2):170-6.

17. Stewart MA. Effective physician-patient communication and health outcomes: a review. Can Med Assn J. 1995;152(9):1423-33.

18. Georgopoulou S, Prothero L, Cruz DP. Physician-patient communication in rheumatology: a systematic review. Rheum Intl. 2018;38:763-75.

19. Centers for Medicare \& Medicaid Services. Accountable Health Communications Model. (https://innovation.cms.gov/initiatives/ahcm). Revised October 2018. Accessed 7 Jan 2019.

20. Alley DE, Asomugha CN, Conway PH, Sanghavi DM. Accountable health communities - addressing social needs through Medicare and Medicaid. N Engl J Med. 2016;374(1):8-11.

21. Fraze $T$, Lewis VA, Rodriguez HP, Fisher ES. Housing, transportation, and food: how ACOs seek to improve population health by addressing nonmedical needs of patients. Hlth Aff. 2016;35(11):2109-15.

22. Association of American Medical Colleges. 1984 medical school graduation questionnaire: all schools report. Washington, DC: Association of American Medical Colleges; 1984.

23. Association of American Medical Colleges. 2011 medical school graduation questionnaire: all schools report. Washington, DC: Association of American Medical Colleges; 2011.

24. Drain PK, Primack A, Hunt DD, Fawzi WW, Holmes KK, Gardner P. Global health in medical education: a call for more training and opportunities. Acad Med. 2007:82(3):226-30.

25. Peluso MJ, Forrestel AK, Hafler JP, Rohrbaugh RM. Structured Global Health programs in U.S. medical schools: a web-based review of certificates, tracks, and concentrations. Acad Med. 2015;88(1):124-30.

26. Drain PK, Mock C, Toole D, et al. Emergence of undergraduate majors in Global Health: systematic review of programs and recommendations for future directions. Am J Trop Med Hyg. 2017;96(1):16-23.

27. Rodriguez MA, Garcia R. First, do no harm: the US sexually transmitted disease experiments in Guatemala. Am J Public Health. 2013;103:2122-6.

28. Whitehead C, Wondimagegn D, Baheretibeb Y, Hodges B. The international partner as invited guest: beyond colonial and import-export models of medical education. Acad Med. 2018;93:1760-3.

29. Spiegel JM, Breilh J, Yassi A. What language matters: insights and challenges in applying a social determination of health approach in a north-south collaborative research program. Glob Health. 2015;11(9):1-17.

30. Duke Global Health Institute. Approaching Global Health through language and culture. (https://globalhealth.duke.edu/media/news/approachingglobal-health-through-language-and-culture). Published 4 Nov 2014. .

31. Morales R, Rodriguez L, Singh A, Stratta E, Mendoza L, Valerio MA, Vela M. National Survey of medical Spanish curriculum in U.S. medical schools. J Gen Intern Med. 2015;30(10):1434-9.

32. O'Brien $M$, Shea J. Disparities in patient satisfaction among Hispanics: the role of language preference. J Immigr Minor Health. 2011;13(2):408-12.

33. Kenison T, Madu A, Krupat E, Ticona L, Vargas IM, Green AR. (2016). Through the veil of language: exploring the hidden curriculum for the Care of Patients with limited English proficiency. Acad Med. 2017;92(1):92-100.

34. Jacobs B, Ryan AM, Henrichs KS, Weiss BD. Medical interpreters in outpatient care. Ann Fam Med. 2018;16:70-6.

35. The National Council on Interpreting in Health Care. A National Code of Ethics For Interpreters in Health Care. 2004. https:/uww.ncihc.org/assets/documents/ publications/NCIHC\%20National\%20Code\%20of\%20Ethics.pdf. Accessed 10 Apr 2019.

36. Flores $\mathrm{G}$. The impact of medical interpreter services on the quality of HealthCare: a systematic review. Med Care Res and Rev. 2005;62(3):255-99.

37. Nápoles AM, Santoyo-Olsson J, Karliner LS, Gregorich SE, Pérez-Stable EJ. Inaccurate language interpretation and its clinical significance in the medical encounters of Spanish-speaking Latinos. Med Care. 2015:53(11):940-7.

38. AAMC. Core Entrustable Professional Activities for Entering Residency: Curriculum Developers' Guide. 2014. https://icollaborative.aamc.org/ resource/887/. Accessed 10 Jan 2019.

39. AAMC. Core Entrustable Professional Activities for Entering Residency: Toolkits for the 13 Core EPAs. 2017. https://www.aamc.org/download/4 82214/data/epa13toolkit.pdf. Accessed 16 Mar 2019.

\section{Publisher's Note}

Springer Nature remains neutral with regard to jurisdictional claims in published maps and institutional affiliations. 